\title{
Lid Restraint Evokes Two Types of Motor Adaptation
}

\author{
Edward J. Schicatano, ${ }^{1}$ Jessica Mantzouranis, ${ }^{2}$ Kavita R. Peshori, ${ }^{2}$ Jill Partin,, ${ }^{3}$ Craig Evinger ${ }^{2}$ \\ ${ }^{1}$ Department of Psychology, Wilkes University, Wilkes-Barre, Pennsylvania 18766, and Departments of ${ }^{2}$ Neurobiology and \\ Behavior and Ophthalmology and ${ }^{3}$ Pediatrics, State University of New York Stony Brook, Stony Brook, New York 11794
}

\begin{abstract}
Unilateral reduction in eyelid motility produced two modes of blink adaptation in humans. The first adaptive modification affected both eyelids. Stimulation of the supraorbital branch of the trigeminal nerve (SO) ipsilateral to the upper eyelid with reduced motility evoked bilateral, hyperexcitable reflex blinks, whereas contralateral SO stimulation elicited normally excitable blinks bilaterally. The probability of blink oscillations evoked by stimulation of the ipsilateral SO also increased with a reduction in lid motility. The increased probability of blink oscillations correlated with the enhanced trigeminal reflex blink excitability. Thus, the trigeminal complex ipsilateral to the restrained eyelid coordinated an increase in excitability and blink oscillations independent of the eyelid experiencing reduced motility. The second type of modification appeared only in the eyelid experiencing reduced motility. When tested immediately after removing lid restraint, blink amplitude increased in this eyelid
\end{abstract}

relative to the normal eyelid regardless of the stimulated SO. A patient with seventh nerve palsy exhibited the same two patterns of blink adaptation. These results were consistent with two forms of adaptation, presumably because unilateral lid restraint produced two error signals. The corneal irritation caused by reduced blink amplitude generated abnormal corneal inputs. The difference between proprioceptive feedback from the blink and expected blink magnitude signaled an error in blink amplitude. The corneal irritation appeared to drive an adaptive process organized through the trigeminal complex, whereas the proprioceptive error signal drove an adaptive process involving just the motoneurons controlling the restrained eyelid.

Key words: motor learning; reflex blinks; facial nerve palsy; motor adaptation; trigeminal; adaptive plasticity
When a movement consistently falls short of its target, the nervous system engages motor learning to modify the programming of subsequent movements to prevent the shortfall. The nervous system can use motor or sensory errors created by the movement shortfall to initiate adaptive motor learning. For example, a sensory error signal arises from the retinal disparity between the target on the retina and the fovea after the initial saccade. The additional saccade necessary to acquire the target creates a motor error signal. Despite the availability of both motor and sensory error signals, the nervous system relies on the visual error to drive saccadic adaptation (Wallman and Fuchs, 1998).

The effect of error signals in driving adaptive motor learning in the trigeminal reflex blink system is poorly understood. Reduced eyelid motility, which produces blink adaptation (Evinger and Manning, 1988; Evinger et al., 1989; Huffman et al., 1996; Baker et al., 1997; Pellegrini and Evinger, 1997), creates two error signals. First, a comparison of trigeminal proprioceptive feedback from the lid movement with the efferent motor signal of the intended blink amplitude creates a blink amplitude error. Second, corneal irritation provides an error signal that indirectly indicates blink size. Because the decreased blink amplitude caused by reduced eyelid motility fails to maintain the tear film adequately (Doane, 1980), activation of corneal afferents by corneal irritation provides an error signal. There are a number of possible roles for

\footnotetext{
Received May 30, 2001; revised Oct. 18, 2001; accepted Oct. 24, 2001.

This work was supported by National Eye Institute Grants EY07391 (C.E.) and EY06808 (E.J.S.). We thank D. Schmidt for technical assistance and I. Kassem, V. M. Henriquez, J.-B. Mao, and A. S. Powers for valuable comments on previous versions of this manuscript.

Correspondence should be addressed to Craig Evinger, Department Neurobiology and Behavior, State University of New York Stony Brook, Stony Brook, NY 11794-5230. E-mail: levinger@notes.cc.sunysb.edu.

Copyright (C) 2002 Society for Neuroscience $\quad 0270-6474 / 02 / 220569-08 \$ 15.00 / 0$
}

these error signals. They could both drive blink adaptation. Each error signal could initiate a different form of adaptation, or one error signal might not participate in driving blink adaptation.

Unilaterally reducing lid motility can distinguish between roles of corneal and proprioceptive error signals in driving trigeminal reflex blink adaptation, because the two error signals should generate different adaptation patterns. The trigeminal complex ipsilateral to the eyelid with reduced motility receives a corneal error signal. This unilateral corneal error signal, however, modifies reflex blinks in both eyelids, because activation of the affected trigeminal complex evokes bilateral blinks in humans (Sibony and Evinger, 1998). Activating the contralateral trigeminal complex, which did not receive a corneal error signal, should evoke normal reflex blinks in both eyelids. Thus, corneal errors should produce blink modifications that are only apparent after activation of the trigeminal complex ipsilateral to the eyelid with reduced motility but affect both eyelids. Previous work implies that a discrepancy between the intended and the actual blink amplitude, the proprioceptive error signal, only modifies the drive on the eyelid experiencing reduced eyelid motility (Pellegrini and Evinger, 1997). Thus, proprioceptive error signals should produce blink modifications that are independent of which trigeminal complex is activated but are only apparent in the affected eyelid. Finally, by comparing the blink modifications produced by unilateral lid restraint in intact subjects with those caused by facial nerve palsy, we can evaluate the role of these error signals in the adaptive processes occurring in this disease state.

\section{MATERIALS AND METHODS}

Five human subjects (two male and three female) 26-52 years of age participated in the study. One female subject (age 34 years) had a facial 
palsy primarily involving the right orbital region caused by reconstructive facial surgery. This subject was treated for dry eye associated with her facial palsy with commercially available drops. None of the remaining subjects had any eyelid disorders or exhibited dry eye symptoms. All subjects gave informed consent for their participation in the study. All experiments were performed in strict accordance with federal, state, and university regulations regarding the use of humans in experiments and received approval of the university Institutional Review Board.

Blink measurement and evocation. Blinks in both eyelids were assessed simultaneously by measuring upper eyelid position, orbicularis oculi EMG (OOemg) activity (for details, see Evinger et al., 1991), or both. To monitor upper eyelid position, a 30 turn coil $(2 \mathrm{~mm}$ diameter, $25 \mathrm{mg}$ weight) was taped to the center of the lower margin of each upper eyelid. Two miniature silver plates $(<2 \mathrm{~mm}$ diameter $)$ were taped to the lateral and medial portions of each upper eyelid to record the pretarsal component of the OOemg activity. Pretarsal OOemg recordings contained significantly less contamination from the activity of other facial muscles, e.g., temporalis and masseter, compared with records of the orbital component of the OOemg activity recorded by the more typical placement of electrodes on the lower eyelid and the lateral canthus. The OOemg signal was amplified and filtered from 0.3 to $2 \mathrm{kHz}$. An electrode on the forehead served as a ground. No subject reported discomfort or exhibited a reduction in lid motility caused by the lid coil or OOemg electrodes.

Electrical stimulation of the supraorbital branch of the trigeminal nerve (SO) evoked trigeminal reflex blinks. Gold-plated cup skin electrodes (Grass Instruments) filled with electrode paste were taped over both the left and right supraorbital nerves, one electrode immediately above the supraorbital notch and the other $2 \mathrm{~cm}$ above the first. The threshold intensity required to evoke the R2 component of SO-evoked blinks was determined for the left and right SO nerve when using a 170 $\mu \mathrm{sec}$ duration stimulus. Supraorbital stimulus intensity was adjusted to twice threshold (2T) intensity for data collection. For all subjects, $2 \mathrm{~T}$ stimulus intensity ranged from 2 to $9 \mathrm{~mA}$. No subject reported these stimuli to be painful or aversive. Only one SO nerve was stimulated at any given time. To maintain a stable level of alertness, subjects watched a videotape during the experiment.

Procedures. Subjects without facial nerve palsy underwent unilateral upper eyelid restraint to impede upper eyelid closure. The following measurements were made: (1) before restraint, control; (2) at 5 min with unilateral lid restraint; (3) at 75 min with lid restraint; (4) at 120 min with lid restraint; (5) at $5 \mathrm{~min}$; and (6) at $30 \mathrm{~min}$ after removing lid restraint. At each of these measurement times, we collected two blocks of 15 trials. In the first block, blinks were evoked by stimulation of the SO ipsilateral to the restrained lid. In the second block, blinks were evoked by stimulation of the SO contralateral to the restrained lid. Each block contained three trial types: (1) five pairs of identical SO stimuli with a $1000 \mathrm{msec}$ interstimulus interval; (2) five pairs of identical SO stimuli with a 500 msec interstimulus interval; and (3) five single SO stimuli. Within each block, the three trial types were intermixed, and a trial was presented every $25 \pm 5 \mathrm{sec}$.

Upper eyelid restraint was achieved with a weight producing an upward force on the upper eyelid. Before beginning the experiment, a 110-mm-long wooden rod was taped on the forehead, parallel to the eyebrow and extending laterally past the temple. At the beginning of lid restraint, a 4-0 silk suture was taped to the center of the upper eyelid at the lower margin. The silk suture was led over the wooden rod and behind the subject's ear, where it was attached to a $9.0 \mathrm{gm}$ weight, which hung down below the earlobe. This restraint did not prevent blinking but initially reduced the downward lid movement to approximately half of that produced by the unrestrained upper eyelid. Because lid restraint interfered with lid coil placement, only OOemg data were collected from the restrained lid during restraint. Lid position and OOemg data were always collected from the contralateral, unrestrained upper eyelid and from both eyelids before and after lid restraint.

For the subject with the right facial nerve palsy, data were collected 30 , $55,93,121$, and $169 \mathrm{~d}$ after the onset of facial palsy. Each experimental session consisted of three blocks of trials. In the first two blocks, the subject received six pairs of identical intensity SO stimuli with interstimulus intervals of $250,500,750$, or $1000 \mathrm{msec}$, for a total of 24 trials. The stimulus pairs were presented in a pseudorandom manner with an intertrial interval of $25 \pm 5 \mathrm{sec}$. The right SO was stimulated in one block, and the left SO was stimulated in the other block. The third block consisted of 10 trials of a single right SO stimulus.

Data collection and analysis. Lid position and OOemg data were
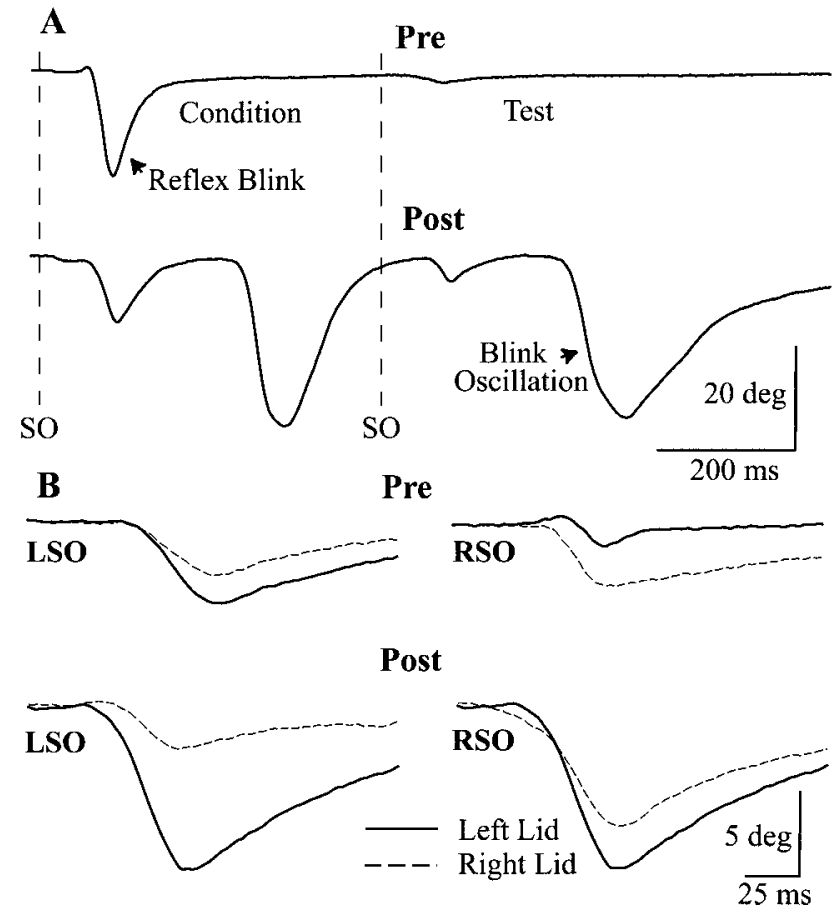

Figure 1. Unilateral lid restraint alters trigeminal reflex blinks. $A$, The relative amplitudes of a blink (Condition) evoked by a $2 \mathrm{~T}$ SO stimulus (dotted line SO) and a blink (Test) elicited by an identical SO stimulus (dotted line SO) $500 \mathrm{msec}$ later are different before (Pre) and after (Post) $165 \mathrm{~min}$ of upper eyelid restraint. After lid restraint, a single 2T SO stimulus evokes a reflex blink and additional blinks (Blink Oscillation) that occur at a constant time relative to the onset of the preceding blink. Each trace is a single trial from the left eyelid. $B$, The relative amplitudes of left lid (solid line) and right lid (dashed line) movement evoked by a left SO (LSO) or right SO (RSO) stimulus are different before (Pre) and after (Post) $2 \mathrm{hr}$ of left upper eyelid restraint. Each trace is a single trial.

digitized at $2 \mathrm{kHz} / \mathrm{channel}$ and stored for off-line analysis. Laboratorydeveloped software allowed the user to determine lid movement amplitude, duration, latency, and maximum velocity for each eyelid. In addition, the user determined the magnitude (integration of the rectified OOemg activity), duration, and latency of OOemg activity.

For each variable, each subject's data were normalized to the median value of the prerestraint, control condition. The data for all subjects were pooled and tested for statistical significance using the Mann-Whitney $U$ rank-sum test or the Wilcoxon signed rank test.

\section{RESULTS}

\section{Effect of unilateral eyelid restraint on trigeminal reflex blink excitability}

Unilateral stimulation of SO elicited a bilateral reflex blink (Fig. $1 A$, top trace). Presentation of pairs of $\mathrm{SO}$ stimuli, the paired stimulus paradigm, demonstrated that the first SO stimulus (Condition) suppressed the blink evoked by the second SO (Test) stimulus. The magnitude of the test/condition ratio was $<1$ for interstimulus intervals of $<1 \mathrm{sec}$ in normal humans (Powers et al., 1997). The paired stimulus paradigm estimated the responsiveness of the trigeminal system to subsequent blink-evoking stimuli after a trigeminal stimulus; i.e., it showed trigeminal reflex blink excitability.

Restraining the eyelid induced a rapid increase in trigeminal reflex excitability evoked by stimulation of the SO ipsilateral to lid restraint (Figs. 1A, bottom trace, 2, O). For the restrained eyelid, reflex blink excitability (test/condition ratio, measured at the 500 msec interstimulus interval) increased significantly relative to 


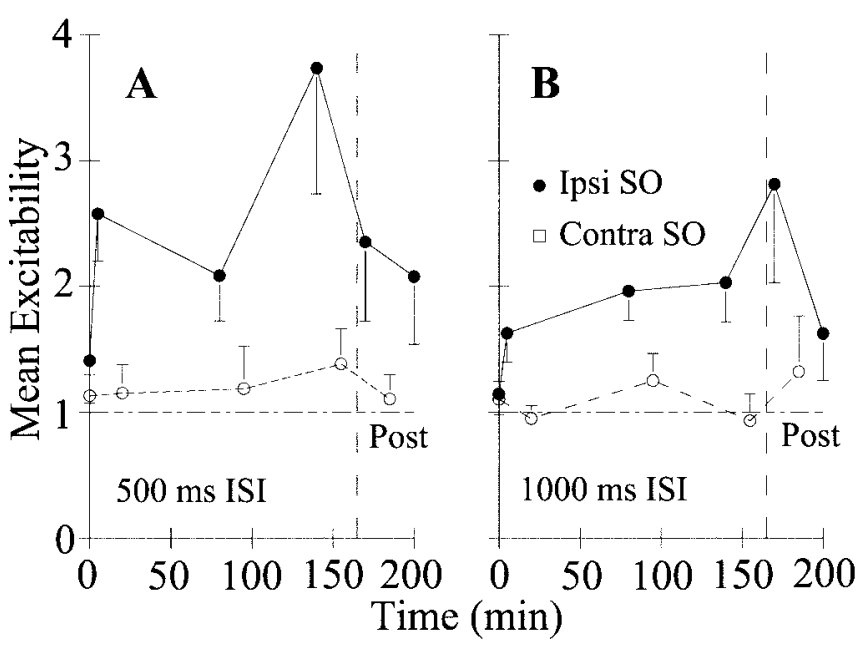

Figure 2. Unilateral lid restraint increases the excitability of blinks evoked by stimulation of the SO ipsilateral (Ipsi) to the restrained eyelid but not to stimulation of the contralateral (Contra) SO. For blinks evoked by the SO ipsilateral $(\bullet)$ and contralateral $(\bigcirc)$ to the restrained eyelid, the mean \pm SEM excitability relative to the median prerestraint excitability for all subjects is plotted as a function of time after unilateral lid restraint for the $500(A)$ and $1000(B)$ msec interstimulus intervals. Lid restraint was removed at $165 \mathrm{~min}$ (Post, dashed line).

prerestraint excitability (Fig. $2 A ; Z_{(15)}=2.76,2.43$, and $2.78 ; p<$ $0.05)$ or relative to the excitability produced by stimulating the $\mathrm{SO}$ contralateral to lid restraint $\left(Z_{(15)}=3.17,2.28\right.$, and 2.03; $p<$ $0.05)$ at all time points. Immediately after removing lid restraint, reflex blinks remained more excitable than prerestraint blinks $\left(Z_{(15)}=1.65 ; p<0.05\right.$; Figs. $1 A, 2 A$, Post $)$. Thirty-five minutes after removing lid restraint, however, excitability returned to prerestraint values $\left(Z_{(15)}=1.22 ; p>0.05\right)$. In contrast to the elevated excitability produced by stimulation of the SO ipsilateral to the restrained eyelid, the excitability after stimulation of the SO contralateral to the restrained lid did not change significantly $\left(Z_{(13)}=0.31,0.59,0.49\right.$, and 0.15; $p>0.05$; Fig. 2, $\left.\bigcirc\right)$. An identical, but not quantitatively as large, pattern of excitability increases occurred for pairs of stimuli with a $1000 \mathrm{msec}$ interstimulus interval (Fig. 2B). Thus, lid restraint rapidly increased the excitability of blinks evoked by stimulation of the SO ipsilateral to the restrained eyelid. This increased excitability was not a simple response to altered feedback from the restrained eyelid, because it remained after removal of lid restraint. Nor was the elevated excitability a generalized response to lid restraint, because stimulation of the contralateral SO always evoked normally excitable blinks.

The excitability exhibited by the restrained and unrestrained eyelids depended on which SO was stimulated rather than which eyelid was measured. For all subjects, the mean excitability measured in the eyelid contralateral to the SO divided by the mean excitability measured in the eyelid ipsilateral to the SO was $0.94 \pm$ 0.1 for the left SO and $1.21 \pm 0.2$ for right SO before lid restraint. Because these values were not significantly different $\left(Z_{(8)}=0.95\right.$; $p>0.05)$, unilateral SO stimulation produced the same excitability in both eyelids. Lid restraint did not alter this relationship. After unilateral lid restraint, the ratio was $1.27 \pm 0.23$ after stimulation of the SO ipsilateral to the restrained eyelid and $0.93 \pm 0.19$ after stimulation of the contralateral SO. These values were not significantly different from each other or prerestraint values $\left(Z_{(8)}=1.05,1.47\right.$, and $\left.1.47 ; p>0.05\right)$. Thus,
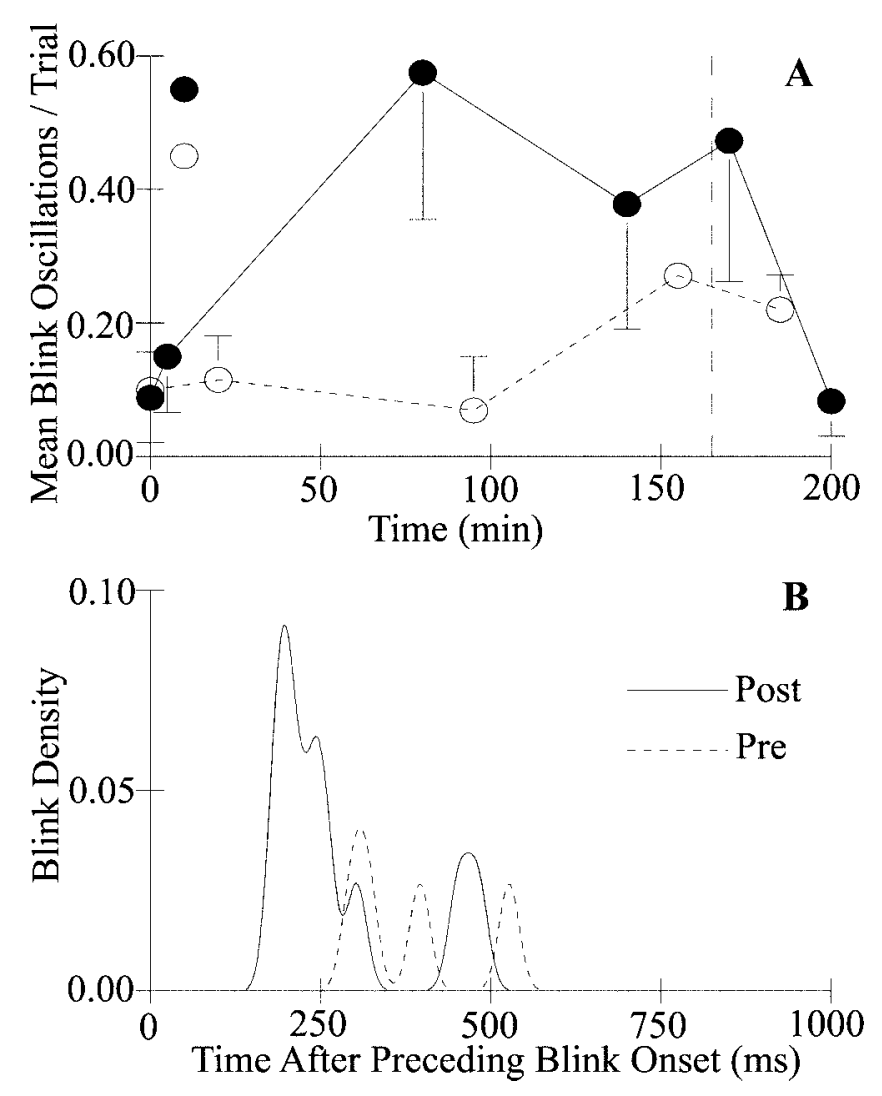

Figure 3. Blink oscillation probability increased and blink oscillation latency decreased with lid restraint. $A$, The mean \pm SEM number of blink oscillations per trial for all subjects is plotted as a function of time after unilateral lid restraint for blink oscillations evoked by the SO ipsilateral (๑) and contralateral $(\bigcirc)$ to the restrained eyelid. Lid restraint was removed at 165 min (dashed line). $B$, Blink density of blink oscillations produced by ipsilateral SO stimulation is plotted as a function of time after the onset of the preceding blink for blink oscillations elicited before (Pre, dashed line) and immediately after (Post, solid line) unilateral lid restraint for a single subject.

regardless of which eyelid was measured, blink excitability depended only on which trigeminal complex was activated.

\section{Effect of unilateral lid restraint on blink oscillations}

Before eyelid restraint, an SO stimulus evoked a single reflex blink (Fig. 1A, Pre). With lid restraint, however, SO stimulation evoked additional blinks that occurred after the reflex blink (Fig. $1 A$, Post, Blink Oscillation). Because these extra blinks occurred at a relatively constant interval with respect to the onset of the preceding blink, these additional blinks were referred to as blink oscillations (Peshori et al., 2001). We estimated the most probable time for a blink oscillation to occur after the onset of the preceding blink by creating a blink density function (Fig. 3B). We replaced the onset time of each blink oscillation relative to the onset of the preceding blink with a $15 \mathrm{msec} \delta$ Gaussian distribution. Because blink oscillations began at similar times relative to the preceding blink, summing these Gaussian distributions created a distribution peaked around the most common time for blink oscillations to occur after the onset of the preceding blink. This distribution provided an estimate of when to expect the occurrence of a blink oscillation after the onset of the preceding blink. To determine the likelihood that a blink oscillation would occur on each trial, we divided the number of blink oscillations by the number of trials for each data block (Fig. $3 A$ ). The amplitude 
of blink oscillations was 1.45 times larger than the condition reflex blink amplitude on average (Fig. $1 B$ ).

Lid restraint significantly increased the probability of blink oscillations (Figs. $1 \mathrm{~A}$, Post, $3 \mathrm{~A}$ ). Before lid restraint, stimulation of the SO ipsilateral or contralateral to the restrained lid evoked a blink oscillation once every 10 or 11 trials (ipsilateral, $0.088 \pm$ 0.07; contralateral, $0.099 \pm 0.06$ blink oscillations per trial; Fig. $3 A, 0, \bigcirc)$. For example, the subject illustrated in Figure $3 \mathrm{~B}$ had four blink oscillations in 14 trials that occurred with almost equal probability between 275 and $525 \mathrm{msec}$ after the onset of the preceding blink (Fig. 3B, Pre). Lid restraint increased the number of blink oscillations evoked by stimulation of the SO ipsilateral to the restrained eyelid for all subjects (Fig. $3 A, 0$ ). The frequency of blink oscillations increased significantly at 80 and 140 min with restraint, and significant elevation continued immediately after release from restraint $\left(Z_{(4)}=1.67,1.67\right.$, and $\left.1.67 ; p<0.05\right)$. For example, immediately after release from lid restraint, stimulation of the SO ipsilateral to the restrained eyelid evoked 10 blink oscillations in 14 trials for the subject illustrated in Figure $3 B$ (Post). The maximum probability of a blink oscillation occurred $197 \mathrm{msec}$ after the onset of the preceding blink. Data from the other subjects showed similar peaked distributions, with maximum values at 138, 139, and $199 \mathrm{msec}$ after the onset of the preceding blink. At $30 \mathrm{~min}$ after release from restraint, blink oscillation frequency returned to prerestraint values $\left(Z_{(4)}=0.18\right.$; $p>0.05)$. A significant increase in the probability of blink oscillations after stimulation of the SO contralateral to the restrained eyelid occurred $155 \mathrm{~min}$ after the beginning of lid restraint and remained elevated $15 \mathrm{~min}$ after release from restraint $Z_{(4)}=1.83$; $p<0.05$; Fig. $3 A, \bigcirc$ ). Although stimulation of the contralateral SO evoked significantly more blink oscillations at the end of the experiment than before restraint (Fig. $3 A, \bigcirc$ ), the peak time of occurrence of blink oscillations was later than that found with ipsilateral SO stimulation. For all subjects, the peak of the blink density distribution after stimulation of the contralateral SO occurred an average of $174.1 \pm 53 \mathrm{msec}$ later than that after ipsilateral SO stimulation. Thus, as with reflex excitability, blink oscillations depended on which trigeminal complex was activated.

\section{Monocular effects of unilateral lid restraint}

Although trigeminal reflex blink excitability and blink oscillation data demonstrated that activation of the trigeminal complex ipsilateral to the restrained eyelid caused binocular adaptive processes, there was also an adaptive modification expressed only by the restrained eyelid. The adaptation appeared as an increased responsiveness of the restrained eyelid to SO stimulation. For the subject illustrated in Figure $1 B$, stimulation of the left SO produced a slightly larger blink in the left (solid line) than in the right (dashed line) eyelid before lid restraint (Fig. 1B, LSO, Pre). Stimulation of the right SO evoked a larger blink in the right eyelid than in the left (Fig. $1 B, R S O$, Pre). For this subject, the average ratio of right lid amplitude/left lid amplitude was 0.81 for left SO stimuli and 2.61 for right SO stimuli. For all subjects, the mean \pm SEM ratios were $0.98 \pm 0.12$ and $1.61 \pm 0.05$. Differences in the amplitude of the two lid movements evoked by SO stimulation are typical for normal subjects (Peshori et al., 2001). Immediately after removing restraint from the left eyelid, however, left SO stimulation evoked a significantly larger blink in the left lid relative to the right lid for the subject in Figure $1 B$ (LSO, Post). In contrast to the Pre data, right SO stimulation elicited nearly equal amplitude blinks in the two eyelids after left lid restraint (Fig. $1 B$, RSO, Post). For this subject, the average ratio of right lid amplitude/left lid amplitude was 0.26 after left SO stimulation and 1.05 after right SO stimulation. For all subjects, restraint significantly increased the amplitude of the previously restrained eyelid relative to the unrestrained eyelid. Stimulation of the SO ipsilateral to the restrained eyelid produced a ratio of $0.47 \pm 0.03$ between the two eyelids, which was significantly less than the ratio before restraint $\left(Z_{(66)}=-6.04 ; p<0.0001\right)$. Stimulation of the SO contralateral to the restrained eyelid produced a ratio of $1.04 \pm 0.06$, which was significantly less than the ratio before restraint $\left(Z_{(1,47)}=3.79 ; p<0.001\right)$. These decreases in the unrestrained lid amplitude/restrained lid amplitude occurred because of the increased blink amplitude of the previously restrained lid relative to the unrestrained lid.

Blink oscillations revealed a similar increase in the responsiveness of the previously restrained eyelid. Regardless of which SO was stimulated, the blink oscillation amplitude increased for the previously restrained eyelid but did not change for the unrestrained eyelid. For all subjects, the mean blink oscillation amplitude before lid restraint/mean blink amplitude after lid restraint was $1.52 \pm 0.16$ for the restrained eyelid but $1.08 \pm 0.04$ for the unrestrained eyelid. The increased amplitude of the blink oscillations in the previously restrained eyelid without an increase in blink oscillation amplitude for the unrestrained eyelid demonstrated that only the motoneurons controlling the restrained eyelid became more responsive with lid restraint. The increased responsiveness of the previously restrained eyelid regardless of which SO was activated further demonstrated that this blink modification occurred only for the motoneurons controlling one eyelid.

\section{Effect of unilateral facial nerve palsy on trigeminal reflex blink excitability}

Short-term mechanical restraint of one eyelid in normal subjects produced a rapid increase in trigeminal reflex blink excitability and blink oscillations in response to stimulation of the trigeminal complex ipsilateral to the restrained eyelid (Figs. $1 A, 2$ ). Unilateral facial nerve palsy may produce a long-term version of this adaptive process. Unlike the adaptive processes set off by mechanical lid restraint, however, blink modifications with facial nerve palsy could also result from pathological changes in the facial nucleus caused by facial nerve damage. If these pathological changes were the primary cause of blink modifications, then only one eyelid should exhibit increased reflex blink excitability regardless of which trigeminal nerve was activated. In contrast, if the blink modifications in trigeminal reflex blink excitability reflected the same adaptive processes as those produced by lid restraint, then which trigeminal complex was activated, rather than which eyelid was measured, should determine reflex blink excitability.

The subject with right facial nerve palsy exhibited increased trigeminal reflex blink excitability after stimulation of the SO ipsilateral to the palsied lid (Figs. 4A, 5). At $30 \mathrm{~d}$ after the onset of a right facial palsy, presentation of pairs of identical stimuli with a $250 \mathrm{msec}$ interval to the SO ipsilateral to the palsied eyelid evoked a much larger blink to the second stimulus than to the first (Fig. 4A, Right SO). The average test/condition blink amplitude ratio at this interval was near 1 (Fig. 5, O), a ratio far greater than that of age-matched control subjects (Fig. 5, $\bullet$ ). In contrast, presentation of the same stimulus pair to the contralateral SO nerve evoked a smaller blink to the second stimulus than to the first (Fig. 4A, Left $S O$ ). Although slightly higher than the excitability of age-matched normal subjects (Fig. 5, $\diamond$ Peshori et al., 


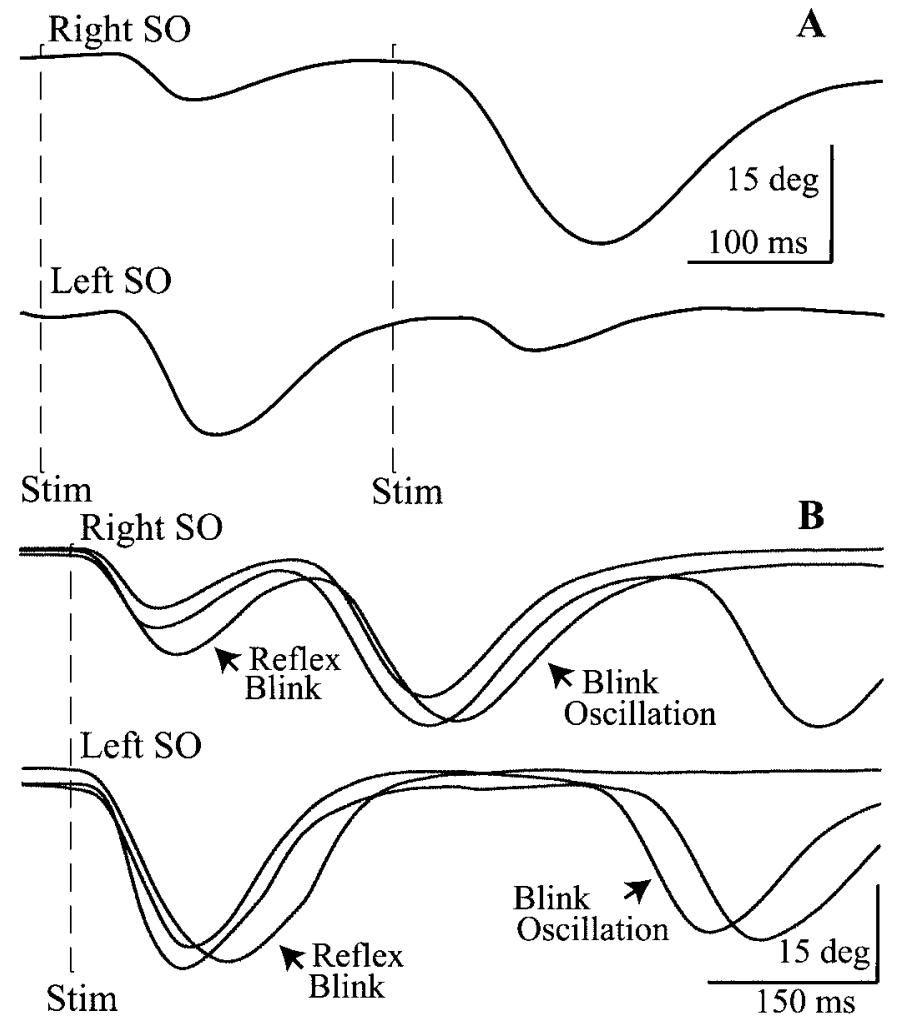

Figure 4. Unilateral facial palsy alters trigeminal blinks. $A$, The relative amplitude of blinks evoked by a 2T SO stimulus (first dashed line Stim) and an identical SO stimulus (second dashed line Stim) occurring $250 \mathrm{msec}$ later are different for stimulation of the SO ipsilateral (Right SO, top trace) or contralateral (Left $S O$, bottom trace) to the right facial palsy. Each trace is a single trial from the unaffected, left, upper eyelid. $B$, A single 2T SO stimulus (dashed line Stim) ipsilateral (Right SO, top traces) and contralateral (Left SO, bottom traces) to the facial palsy evokes a reflex blink and additional blinks (Blink Oscillation) that occur at a constant time relative to the onset of the preceding blink. Each trace is a single trial from the unaffected, left, upper eyelid.

2001), the average test/condition blink amplitude ratio was $<1$ after contralateral SO stimulation (Fig. 5, ○). Thus, the modification in excitability produced by facial palsy depended on which trigeminal complex was activated. Trigeminal reflex blink excitability varied with the interstimulus interval of the stimulus pairs and the time after the onset of facial palsy (Fig. 5). For example, $30 \mathrm{~d}$ after facial palsy onset, $250 \mathrm{msec}$ paired stimulation of the SO ipsilateral to the lid palsy generated much greater excitability than the same stimulus pair presented $121 \mathrm{~d}$ after the onset of facial palsy (Fig. 5, O, $\mathbf{\Delta}$ ). In contrast, the excitability at the 1000 msec interstimulus interval was similar at these two time points.

The excitability of the trigeminal blink reflex depended on the motility of the palsied lid. We estimated lid motility of the palsied eyelid by calculating the ratio of the palsied eyelid blink amplitude to that of the unaffected eyelid during a blink. During the $169 \mathrm{~d}$ over which the subject was tested, this ratio rose from 0.14 to 0.82 . Plotting the ratio of excitability evoked by SO stimulation ipsilateral to the palsied lid relative to the excitability of age-matched control subjects as a function of palsied eyelid motility revealed that excitability decreased as motility recovered for $250 \mathrm{msec}$ paired SO stimuli (Fig. 6, O). When the ratio of palsied and unaffected eyelid amplitude reached 0.82 , blink excitability approached normal values and was nearly the same for both left and right SO stimulation. Thus, a decrease in trigeminal reflex blink

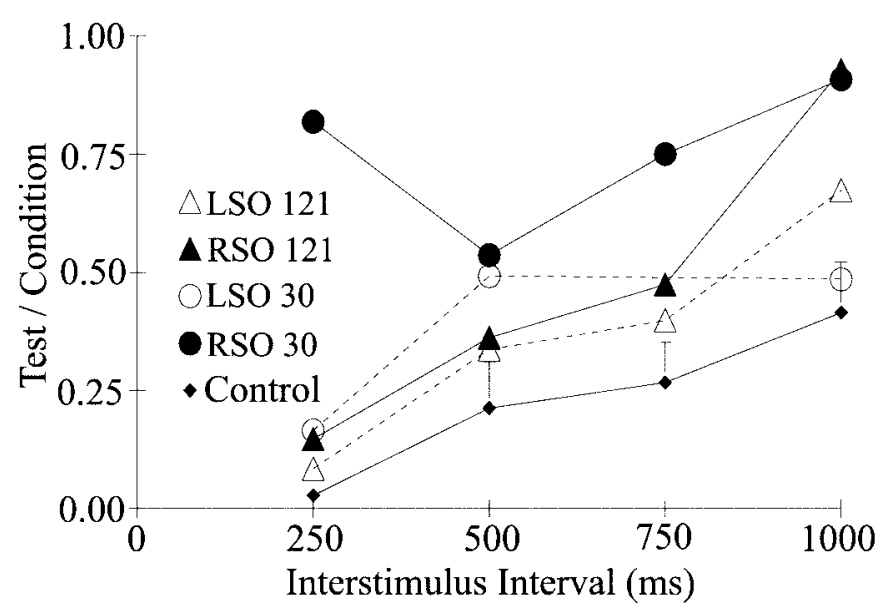

Figure 5. Trigeminal reflex blinks of the subject with right facial nerve palsy were hyperexcitable relative to those of age-matched control subjects. For the unaffected eyelid, the amplitude of the blink evoked by the second of two identical SO stimuli (Test) divided by the amplitude of the blink evoked by the first SO stimulus (Condition) is plotted as a function of the interstimulus interval between the SO stimuli for age-matched control subjects (Control, $\downarrow$; $\mathrm{SEM}$ ) and the facial palsy subject tested 30 $(\boldsymbol{\bullet}, \bigcirc)$ and $121(\boldsymbol{\Lambda}, \triangle)$ d after the onset of right facial nerve palsy. $\boldsymbol{\bullet}, \boldsymbol{\Delta}$, Data from stimulation of the SO ipsilateral to the facial palsy $(R S O)$. $O$, $\triangle$, Data from stimulation of the SO contralateral to the facial palsy $(L S O)$. Each point is the mean of at least four trials. Control subject data are from those of Peshori et al. (2001).

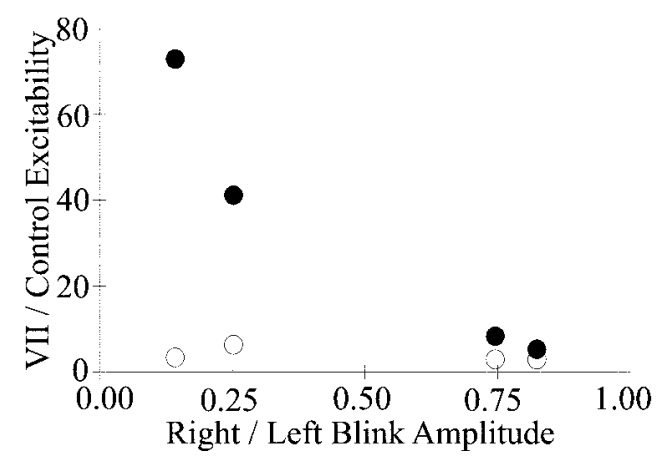

Figure 6. Eyelid motility determines trigeminal reflex blink excitability. The mean excitability of the subject with right facial nerve palsy divided by the mean excitability of age-matched control subjects for the $250 \mathrm{msec}$ interstimulus interval is plotted as function of the mean blink amplitude of the palsied eyelid divided by the mean blink amplitude of the unaffected eyelid for each day tested. $\bullet$, Data from stimulation of the SO ipsilateral to the right facial nerve palsy (right SO). $\bigcirc$, Data from stimulation of the contralateral SO (left SO).

excitability accompanied the recovery of motility by the palsied eyelid.

\section{Effect of unilateral facial palsy on blink oscillations}

The subject with facial nerve palsy experienced dry eye caused by incomplete closure of the right eyelid. The dry eye symptoms improved with the recovery of right eye motility and treatment with eye drops. As typically occurs with dry eye (Evinger et al., 1997a,b), SO stimulation of the facial palsy patient evoked blink oscillations in addition to reflex blinks (Fig. 4B). The typical latency of blink oscillations relative to the onset of the preceding blink depended on which SO nerve was stimulated (Figs. 4B, 7A). At $30 \mathrm{~d}$ after the onset of facial nerve palsy, stimulation of the SO ipsilateral to the palsied eyelid evoked an average of 1.21 blink oscillations per trial that occurred most frequently $189 \mathrm{msec}$ after 


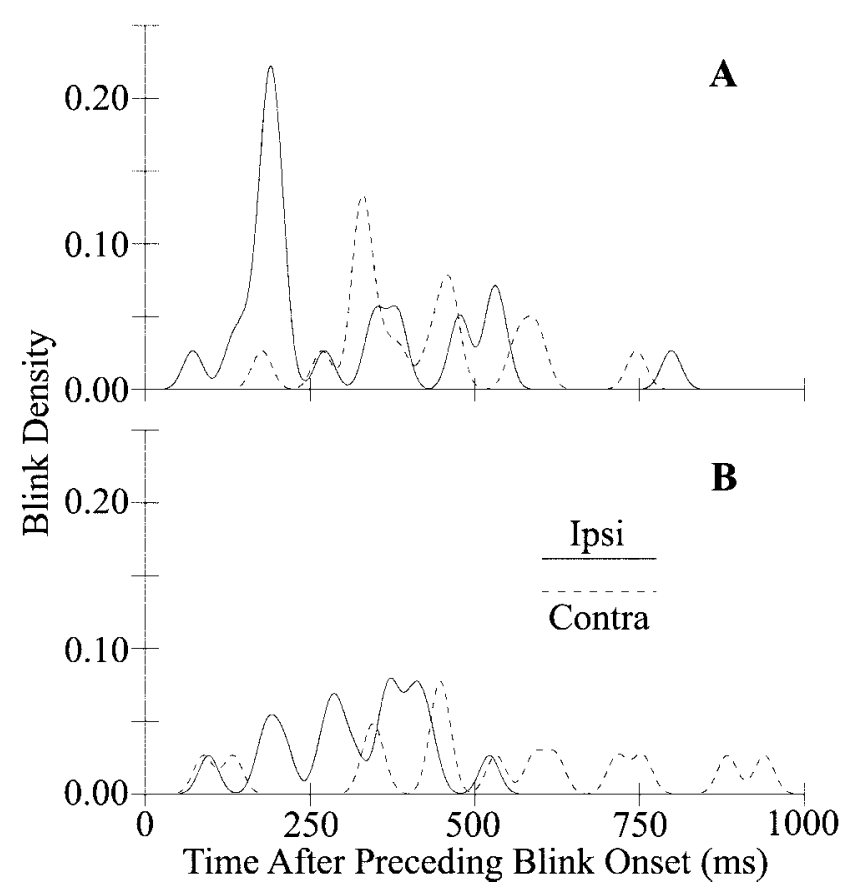

Figure 7. The timing of blink oscillations is different after stimulation of the SO ipsilateral (Ipsi, solid traces) and contralateral (Contra, dashed traces) to the eyelid with facial nerve palsy. Blink density is plotted as a function of time relative to the onset of the preceding blink for blink oscillations elicited by stimulation of the ipsilateral and contralateral SO $30(A)$ and $93(B)$ d after the onset of facial nerve palsy.

to the onset of the preceding blink (Fig. $7 A$, solid trace). Stimulation of the $\mathrm{SO}$ contralateral to the facial palsy evoked an average of 1.13 blink oscillations per trial with a peak blink oscillation latency of $333 \mathrm{msec}$ (Fig. 7A, dashed trace). Although activation of both the left and right trigeminal complexes produced blink oscillations, the two complexes produced oscillations at different times relative to the onset of the preceding blink. During the first 2 months of facial palsy, stimulation of the contralateral SO produced broader latency distributions than did ipsilateral SO stimulation. At $121 \mathrm{~d}$ after the onset of facial nerve palsy, however, right and left SO stimuli evoked an average of 0.48 and 0.45 blink oscillations per trial with broad latency distributions peaking at 376 and $447 \mathrm{msec}$, respectively (Fig. 7B). Thus, blink oscillation frequency decreased and its peak latency increased and became less consistent with the decrease in dry eye symptoms and concurrent corneal irritation.

Both unilateral lid restraint and seventh nerve palsy produced a correlated increase in trigeminal reflex blink excitability and the frequency of blink oscillations (Fig. 8). The higher the blink excitability relative to excitability before lid restraint (Fig. 8, $\square$ ) or relative to age-matched controls (Fig. 8, $\mathbf{\square}$ ), the more blink oscillations per trial a subject exhibited.

\section{DISCUSSION}

\section{Two modes of blink modification}

The data revealed two different patterns of blink adaptation in response to a unilateral reduction in lid motility. The first mode was specific to activation of the trigeminal complex ipsilateral to the restrained eyelid. Stimulation of the SO ipsilateral to the eyelid with reduced motility elicited hyperexcitable trigeminal reflex blinks in both eyelids, whereas contralateral SO stimulation evoked normally excitable or less excitable trigeminal reflex blinks

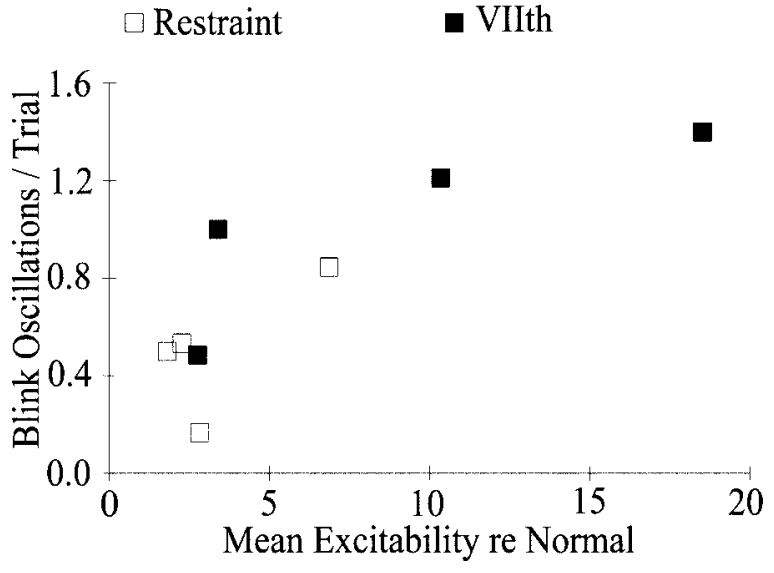

Figure 8 . Trigeminal reflex blink excitability correlates with blink oscillations per trial. Blink oscillations per trial are plotted as a function of trigeminal reflex blink excitability averaged over all tested intervals in the paired stimulus paradigm relative to prerestraint data $(\square)$ or relative to data from age-matched control subjects ( $\square$; Peshori et al., 2001). Each open symbol indicates the average data from one subject with lid restraint, and each filled symbol indicates data from $1 \mathrm{~d}$ of testing of the seventh nerve palsy subject.

in both eyelids (Figs. 2, 5, 6) (Manca et al., 2001). In addition, stimulation of the SO ipsilateral to the eyelid with reduced motility elicited more frequent and shorter-latency blink oscillations than stimulation of the contralateral SO (Figs. $3 A, 4,7$ ). The correlated increase in blink oscillation frequency with reflex blink excitability (Fig. 8) suggests that these modifications were two expressions of the same adaptive process. The second mode of blink adaptation was specific to the eyelid experiencing reduced motility. Relative to the blink made by the contralateral eyelid, SO stimuli evoked a larger blink in the previously restrained eyelid after lid restraint (Fig. $1 B$ ) regardless of which trigeminal complex was activated. After lid restraint, blink oscillation amplitude did not change in the unrestrained eyelid, but the previously restrained eyelid exhibited a $52 \%$ increase in amplitude.

Although either corneal irritation or proprioceptive error signals may be able to initiate both of these adaptive modifications, it appears more likely that each error signal initiates only one mode of adaptation. The adaptation specific to activation of the trigeminal complex ipsilateral to the eyelid with reduced motility effectively compensates for the tear film disruption that causes corneal irritation. The increased trigeminal sensitivity revealed by reflex blink hyperexcitability can result in more frequent reflex blinks. The development of blink oscillations produces larger and more frequent blinks than reflex blinks alone (Figs. 1, 4). The increase in blink amplitude and frequency reduces tear film breakup by spreading tears and increasing the meibomian oil excreted to stabilize the aqueous component of the tear film (Doane, 1980, 1981; Nakamori et al., 1997; Bron and Tiffany, 1998). Despite their efficacy in reducing corneal irritation, these adaptive modifications do not alleviate the proprioceptive error signal created by the difference in actual and intended blink amplitude. The augmented responsiveness of motoneurons innervating the restrained eyelid, however, compensates for the reduced eyelid motility that creates the proprioceptive error. Thus, it appears that corneal irritation initiates a compensatory increase in trigeminal excitability and blink oscillations, whereas the proprioceptive error signal enhances the responsiveness of the motoneurons innervating one eyelid. 


\section{Facial nerve palsy and lid restraint produce the same blink adaptations}

Although corneal irritation-induced modifications involving the trigeminal complex ipsilateral to the palsied lid are qualitatively identical for facial palsy and lid restraint, facial palsy also modifies the contralateral trigeminal complex. The involvement of the contralateral trigeminal complex probably reflects the intense and long-lasting sensory error signal created by facial palsy rather than the occurrence of a unique blink modification associated with facial palsy. The extreme hyperexcitability of the trigeminal complex ipsilateral to the palsied lid can create an abnormal input to the contralateral trigeminal complex in two ways. First, the increased drive onto both the palsied and the normal facial nuclei from the trigeminal complex ipsilateral to the palsied lid causes the normal eyelid to make larger and more frequent blinks than necessary to maintain a normal tear film (Sibony et al., 1991; Huffman et al., 1996). This excessive blinking can remove tears too rapidly (Doane, 1980, 1981), creating a mild corneal irritation in the unaffected eye (Spiera et al., 1997; Sahlin et al., 2000). The corneal irritation of the unaffected eyelid would initiate hyperexcitability and blink oscillations in the trigeminal complex contralateral to the palsied eyelid. The long-lasting corneal irritation produced by facial palsy could also affect the contralateral trigeminal nucleus via direct projections between the two trigeminal complexes (Warren et al., 1997). A recent report confirms the importance of the duration of facial palsy symptoms in modifying the excitability of the contralateral trigeminal complex. Syed et al. (1999) reported that subjects with incomplete recovery from unilateral facial nerve palsy exhibited hyperexcitable trigeminal reflex blinks after stimulation of the SO contralateral to the palsied eyelid, but that subjects with complete recovery exhibited normal trigeminal reflex blink excitability.

Although the weakness of the palsied eyelid masks the increases in blink amplitude that reveal the enhanced motoneuron responsiveness apparent with lid restraint, facial palsy also augments the responsiveness of motoneurons innervating the palsied eyelid. Evidence of the increased responsiveness of orbicularis oculi motoneurons surviving facial nerve damage is the appearance of an R1 response evoked by contralateral SO stimulation (Bratzlavsky and vander Eecken, 1977; Nacimiento et al., 1992). In addition, Cossu et al. (1999) present electrophysiological evidence of hyperexcitability of orbicularis oculi motoneurons recovering from facial palsy. Thus, the long duration and intensity of a unilateral reduction in lid motility produced by facial nerve palsy amplifies both modes of adaptation engendered by lid restraint.

\section{Neural mechanisms underlying the two adaptive processes}

Interactions among the cerebellum, orbicularis oculi motoneurons, and the trigeminal complex probably are sufficient to account for the two modes of blink adaptation caused by a unilateral reduction in lid motility. The occurrence of blink adaptation in decerebrate animals (Evinger et al., 1989; Pellegrini and Evinger, 1997) demonstrates that blink adaptation does not require neural structures rostral to the superior colliculus.

Because the trigeminal complex coordinately regulates reflex blink excitability and blink oscillations (Fig. 8), this structure appears to be critical in the adaptive response to corneal irritation. Increased blink excitability with lid restraint probably results from a change in baseline activity caused by decreased inhibition or enhanced excitatory drive. For example, decreasing nucleus raphe magnus inhibition of the trigeminal complex produces the increased trigeminal reflex blink excitability of Parkinson's disease (Basso et al., 1993, 1996; Basso and Evinger, 1996). Corneal irritation may increase excitability by reducing spinal trigeminal caudalis subdivision inhibition of trigeminal primary afferents that activate reflex blink circuits (Scibetta and King, 1969; Pellegrini and Evinger, 1995; Meng et al., 1997; Henriquez and Evinger, 2000; Hirata et al., 2000). This modification could enhance the excitatory drive onto trigeminal blink circuits by trigeminal primary afferents. Although the neural basis of blink oscillations is unknown, it may involve convergence of innocuous A-fiber and corneal afferents on wide dynamic range neurons. These neurons are a major component of the trigeminal reflex blink circuit that generates SO-evoked blinks (Ellrich and Treede, 1998). Corneal irritation may alter the threshold characteristics of wide dynamic range neurons so that an innocuous input activates blink oscillations as well as a reflex blink. Recent data suggest that modifying these neurons alters blink reflex motor learning in humans (Mao and Evinger, 2001). Thus, the trigeminal complex may be a site of motor learning as well as a critical element for the expression of motor learning.

Considerable evidence suggests that the cerebellum modifies the responsiveness of orbicularis oculi motoneurons. Via the red nucleus, and possibly other structures, the interpositus nucleus modulates orbicularis oculi motoneuron activity (Bracha and Bloedel, 1996; Gruart et al., 1997). Inactivation of the red (Chapman et al., 1990; Bracha et al., 1993) or the interpositus nuclei (Welsh and Harvey, 1989; Hesslow, 1994) reduces the trigeminal reflex blink amplitude. Conversely, interpositus and red nucleus stimulations excite facial motoneurons (Fanardjian and Manvelyan, 1984; Ivarsson and Hesslow, 1993). Finally, lesioning the blink-related cerebellar cortex blocks adaptive increases in blink magnitude with lid restraint (Pellegrini and Evinger, 1997). The error signal created by the difference between the proprioceptive feedback from the actual lid movement and the intended blink must adjust Purkinje cell and interpositus neuron activity to modify the responsiveness of the ipsilateral orbicularis oculi motoneurons.

\section{REFERENCES}

Baker RS, Sun WS, Hasan SA, Rouholiman BR, Chuke JC, Cowen DE, Porter JD (1997) Maladaptive neural compensatory mechanisms in Bell's palsy-induced blepharospasm. Neurology 49:223-229.

Basso MA, Evinger C (1996) An explanation for reflex blink hyperexcitability in Parkinson's disease: II. Nucleus raphe magnus. J Neurosc 16:7318-7330.

Basso MA, Strecker RE, Evinger C (1993) Midbrain 6-hydroxydopamine lesions modulate blink reflex excitability. Exp Brain Res 94:88-96.

Basso MA, Powers AS, Evinger C (1996) An explanation for reflex blink hyperexcitability in Parkinson's disease: I. Superior colliculus. J Neurosci 16:7308-7317.

Bracha V, Bloedel JR (1996) The multiple-pathway model of circuits subserving the classical conditioning of withdrawal reflexes. In: The acquisition of motor behaviors in vertebrates (Bloedel JR, Ebner TJ Wise SP, eds), pp 175-204. Cambridge, MA: MIT.

Bracha V, Stewart SL, Bloedel JR (1993) The temporary inactivation of the red nucleus affects performance of both conditioned and unconditioned nictitating membrane responses in the rabbit. Exp Brain Res 94:225-236.

Bratzlavsky M, vander Eecken H (1977) Altered synaptic organization in facial nucleus following facial nerve regeneration: an electrophysiological study in man. Ann Neurol 2:71-73.

Bron AJ, Tiffany JM (1998) The meibomian glands and tear film lipids. Structure, function and control. Adv Exp Med Biol 438:281-295.

Chapman PF, Steinmetz JE, Sears LL, Thompson RF (1990) Effects of lidocaine injections in the interpositus nucleus and red nucleus on conditioned behavioral and neural responses. Brain Res 537:149-156.

Cossu G, Valls-Solé J, Valldeoriola F, Mun̆oz E, Benĩtez P, Aguilar F 
(1999) Reflex excitability of facial motoneurons at onset of muscle reinnervation after facial nerve palsy. Muscle Nerve 22:614-620.

Doane MG (1980) Interaction of eyelids and tears in corneal wetting and the dynamics of the normal human eyeblink. Am J Ophthalmol 89:507-516.

Doane MG (1981) Blinking and the mechanics of the lacrimal drainage system. Ophthalmology 88:844-851.

Ellrich J, Treede R-D (1998) Characterization of blink reflex interneurons by activation of diffuse noxious inhibitory controls in man. Brain Res 803:161-168.

Evinger C, Manning KA (1988) A model system for motor learning: adaptive gain control of the blink reflex. Exp Brain Res 70:527-538.

Evinger C, Pellegrini JJ, Manning KA (1989) Adaptive gain modification of the blink reflex: a model system for investigating the physiological bases of motor learning. Ann NY Acad Sci 563:87-100.

Evinger C, Manning KA, Sibony PA (1991) Eyelid movements: mechanisms and normal data. Invest Ophthalmol Vis Sci 32:387-400.

Evinger C, Peshori KR, Sibony PA, Wittpenn J (1997a) "Blink oscillations" associated with dry eye. Invest Ophthalmol Vis Sci 38:S117.

Evinger C, Schicatano EJ, Peshori KR, Henriquez VM, Sibony PA, Walcott B (1997b) Oscillations of blinking: an eye on "dry eye." Soc Neurosci Abstr 23:759.

Fanardjian VV, Manvelyan LR (1984) Peculiarities of cerebellar excitation of facial motoneurons. Neurosci Lett 49:265-270.

Gruart A, Pastor AM, Armengol JA, Delgado-García JM (1997) Involvement of cerebellar cortex and nuclei in the genesis and control of unconditioned and conditioned eyelid motor responses. Prog Brain Res 114:511-528.

Henriquez VM, Evinger C (2000) Microstimulation in the caudal trigeminal nucleus modifies corneally evoked reflex blinks. Soc Neurosci Abstr 26:1992.

Hesslow G (1994) Inhibition of classically conditioned eyeblink responses by stimulation of the cerebellar cortex in the decerebrate cat. J Physiol (Lond) 476:245-256.

Hirata H, Takeshita S, Hu JW, Bereiter DA (2000) Cornea-responsive medullary dorsal horn neurons: modulation by local opioids and projections to thalamus and brain stem. J Neurophysiol 84:1050-1061.

Huffman MD, Baker RS, Stava MW, Chuke JC, Rouholiman BR, Porter JD (1996) Kinematic analysis of eyelid movements in patients recovering from unilateral facial nerve palsy. Neurology 46:1079-1085.

Ivarsson M, Hesslow G (1993) Bilateral control of the orbicularis oculi muscle by one cerebellar hemisphere in the ferret. NeuroReport 4:1127-1130.

Manca D, Mun̆oz E, Pastor P, Valldeoriola F, Valls-Solé J (2001) Enhanced gain of blink reflex responses to ipsilateral supraorbital afferent inputs in patients with facial nerve palsy. Clin Neurophysiol 112:153-156.

Mao J-B, Evinger C (2001) Long-term potentiation of the human blink reflex. J Neurosci 21:RC1511-4.
Meng ID, Hu JW, Benetti AP, Berieiter DA (1997) Encoding of corneal input in two distinct regions of the spinal trigeminal nucleus in the rat: cutaneous receptive field properties, responses to thermal and chemical stimulation, modulation by diffuse noxious inhibitory controls, and projections to the parabrachial area. J Neurophysiol 77:43-56.

Nacimiento W, Podoll K, Graeber MB, Topper R, Mobius E, Ostermann H, Noth J, Kreutzberg GW (1992) Contralateral early blink reflex in patients with facial nerve palsy: indication for synaptic reorganization in the facial nucleus during regeneration. J Neurol Sci 109:148-155.

Nakamori K, Odawara M, Nakajima T, Mitzutani T, Tsubota K (1997) Blinking is controlled primarily by ocular surface conditions. Am J Ophthalmol 124:24-30.

Pellegrini JJ, Evinger C (1995) The trigeminally evoked blink reflex. II. Mechanisms of paired-stimulus suppression. Exp Brain Res 107:181-196.

Pellegrini JJ, Evinger C (1997) Role of the cerebellum in adaptive modification of reflex blinks. Learn Mem 3:77-87.

Peshori KR, Schicatano EJ, Gopalaswamy R, Sahay E, Evinger C (2001) Aging of the trigeminal blink system. Exp Brain Res 136:351-363.

Powers AS, Schicatano EJ, Basso MA, Evinger C (1997) To blink or not to blink: inhibition and facilitation of reflex blinks. Exp Brain Res 113:283-290

Sahlin S, Chen E, Kaugesaar T, Almqvist H, Kjellberg K, Lennerstrand $\mathrm{G}$ (2000) Effect of eyelid botulinum toxin on lacrimal drainage. Am J Ophthalmol 129:481-486.

Scibetta CJ, King RB (1969) Hyperpolarizing influence of trigeminal nucleus caudalis on primary afferent preterminals in trigeminal nucleus oralis. J Neurophysiol 33:229-238.

Sibony PA, Evinger C (1998) Anatomy and physiology of normal and abnormal eyelid position and movement. In: Walsh and Hoyt's clinical neuro-ophthalmology, Ed 5 (Miller NR, Newman NJ, eds), pp 15091592. Baltimore: Williams \& Wilkins.

Sibony PA, Evinger C, Manning KA (1991) Eyelid movements in facial paralysis. Arch Ophthalmol 109:1555-1561.

Spiera H, Asbell PA, Simpson DM (1997) Botulinum toxin increases tearing in patients with Sjögren's syndrome: a preliminary report. J Rheumatol 24:1842-1843.

Syed NA, Delgado A, Sandbrink F, Schulman AE, Hallett M, Floeter MK (1999) Blink reflex recovery in facial weakness: an electrophysiologic study of adaptive changes. Neurology 52:834-838.

Wallman J, Fuchs AF (1998) Saccadic gain modification: visual error drives motor adaptation. J Neurophysiol 80:2405-2416.

Warren S, Andrew DL, May PJ (1997) Morphology of intratrigeminal cells and axons. Soc Neurosci Abstr 23:152.

Welsh JP, Harvey JA (1989) Cerebellar lesions and the nictitating membrane reflex: performance deficits of the conditioned and unconditioned response. J Neurosci 9:299-311. 